SCIENTIFIC REPORT

\title{
Expression of protein gene product 9.5 in lepromatous eyes showing ciliary body nerve damage and a "dying back" phenomenon in the posterior ciliary nerves
}

\author{
G J Ebenezer, E Daniel
}

Br J Ophthalmol 2004;88:178-181. doi: 10.1136/bjo.2003.027276

Background/Aim: Peripheral nerve destruction is the hallmark of leprosy. Ocular complications form a substantial part of the clinical manifestations but histopathology of nerve destruction within ocular structures has not been shown satisfactorily. The role of protein gene product (PGP) 9.5 in identifying nerve destruction in the ciliary body and posterior ciliary nerves of lepromatous eyes is shown.

Methods: Serial sections from two lepromatous eyes and two non-lepromatous eyes were stained with PGP 9.5. Histopathological comparison was done on the expression of the PGP 9.5 stain in nerves within the ciliary body, posterior ciliary nerves adjacent to the optic nerve, and nerves tracking through the sclera.

Results: In non-lepromatous eyes, PGP 9.5 was expressed in nerves within the ciliary body, the nerves within the sclera, and posterior ciliary nerves adjacent to the optic nerve. In lepromatous eyes no PGP 9.5 was expressed, signifying nerve destruction.

Conclusions: Nerve destruction in lepromatous eyes has been confirmed histopathologically by the absence of or patchy staining with PGP 9.5. Nerve destruction in the ciliary body can extend to the posterior ciliary nerves by an ascending axonopathy. This "dying back" phenomenon is akin to the "glove and stocking" anaesthesia found in lepromatous leprosy.

$\mathrm{D}$ espite intensive efforts at eradication, leprosy is still endemic in certain countries. An estimated 700000 new cases are diagnosed annually worldwide. ${ }^{1}$ Numerous clinical reports have documented ocular complications that occur in leprosy but few studies corroborate the histopathological process underlying these clinical manifestations..$^{2-5}$ The basic pathology in all forms of leprosy is inflammation and destruction of peripheral nerves. Destruction of peripheral nerves is easily demonstrable in routine skin and nerve biopsies but nerve destruction within ocular tissues has not been well documented. We studied the histopathological changes in the nerves of the ciliary body, nerves within the sclera, and the posterior ciliary nerves adjacent to the optic nerve in two enucleated lepromatous eyes using the neuronal marker protein gene product 9.5 (PGP 9.5).

\section{METHODS}

Two intact eyeballs enucleated from two lepromatous leprosy patients were available for histopathology. One was a painful, blind, left eye from a 40 year old lepromatous leprosy (LL) patient who manifested the disease at 15 years of age and received 25 years of Dapsone monotherapy. He had impaired corneal sensation, chronic iridocyclitis and no perception of light in both eyes, corneal opacities and a limbal leprosy nodule in the right eye, and lagophthalmos in the left eye. Skin smears were positive for acid fast bacilli and his limbs were anaesthetic. The right eye was enucleated after repeated requests and written consent from the patient. The other eye was from a LL patient from a distant leprosy hospital, clinical details of whom were few. Two other eyeballs, both painful blind eyes, enucleated from non-leprosy patients were used as controls.

The eye specimens were fixed in $10 \%$ buffered formalin. Sagittal slab sections were taken, processed, and embedded in paraffin. Five micron thick serial sections were stained with haematoxylin and eosin, modified Fite Faraco stain for acid fast bacilli and Solochrome cyanine stain for myelin. Immunohistochemical staining was done with polyclonal antibody to neuron specific ubiquitin hydrolase, PGP 9.5.

\section{IMMUNOHISTOCHEMISTRY}

Five micron sections were cut and loaded on poly-L-Lysine coated slides and kept at $37^{\circ} \mathrm{C}$ overnight before staining. After de-paraffinisation, antigen retrieval was done by heating the slides at $100^{\circ} \mathrm{C}$ for five minutes in distilled water using a micro-oven. The slides were rinsed in TRIS buffered saline (TBS) ( $\mathrm{pH} 7.4$ ), bleached to remove iris pigments by soaking in $0.25 \%$ potassium permanganate for 15 minutes, rinsed again in TBS and washed in 5\% oxalic acid for two minutes. Blocking was then done with $0.4 \%$ triton-X 100 and $3 \%$ normal serum (NS) in TBS buffer. The sections were incubated at $4^{\circ} \mathrm{C}$ overnight with polyclonal antibody to human PGP 9.5 (1:1200, Vector Labs, Burlingame, CA, USA) suspended in $0.1 \%$ Triton-X 100 and 3\% normal serum in TBS. The sections were rinsed in TBS and incubated in secondary antibody for one hour at room temperature (goat anti-rabbit IgG, diluted $1: 200$ in $0.1 \%$ Triton-X $100,3 \%$ NS in TBS buffer). Sections were then washed in TBS and endogenous peroxidase was blocked with $0.5 \% \mathrm{H}_{2} \mathrm{O}_{2}$ in methanol and rinsed in TBS. The sections were then incubated with AvidinBiotin Complex solution (Vector Labs) for one hour at room temperature followed by rinsing in TBS. The sections were then incubated in vector SG peroxidase substrate (Vector SG substrate kit for peroxidase, Vector Labs) made in phosphate buffered saline, (PBS pH 7.5) for 5-6 minutes for development of chromogen reaction and rinsed again in PBS. Sections were then counter stained with eosin.

\section{RESULTS}

Control eyes I and II

Staining of the nerve twigs within the ciliary muscles with the PGP 9.5 was easily visualised (fig 1A). There was intense

Abbreviations: LL, lepromatous leprosy; PGP, protein gene product; TBS, TRIS buffered saline 

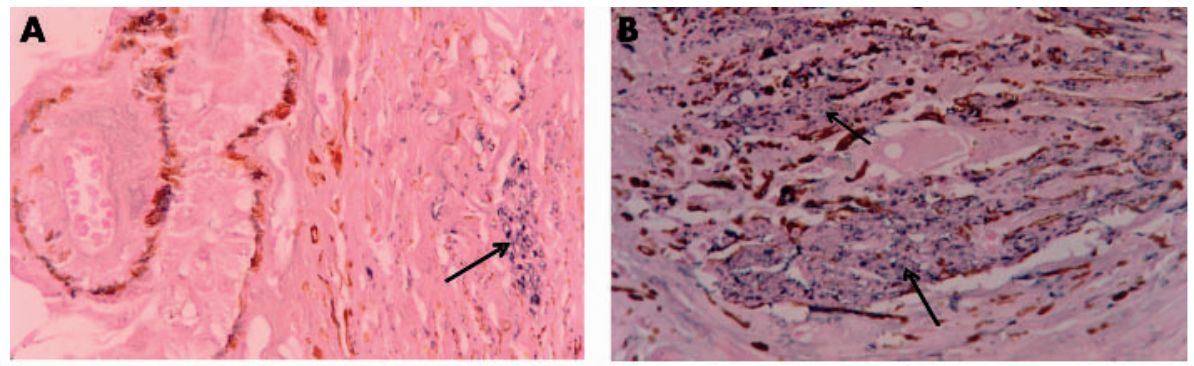

Figure 1 Non-leprosy, control eyes, histopathology. (A) Photomicrograph of ciliary muscle showing healthy ciliary body nerve endings

(immunohistochemical stain PGP $9.5 \times 400)$ ). (B) Photomicrograph of ciliary muscle showing intensely staining ciliary body nerves (immunohistochemical stain PGP $9.5 \times 400$ ). (C) Photomicrograph showing intense staining of the posterior ciliary nerves and ciliary
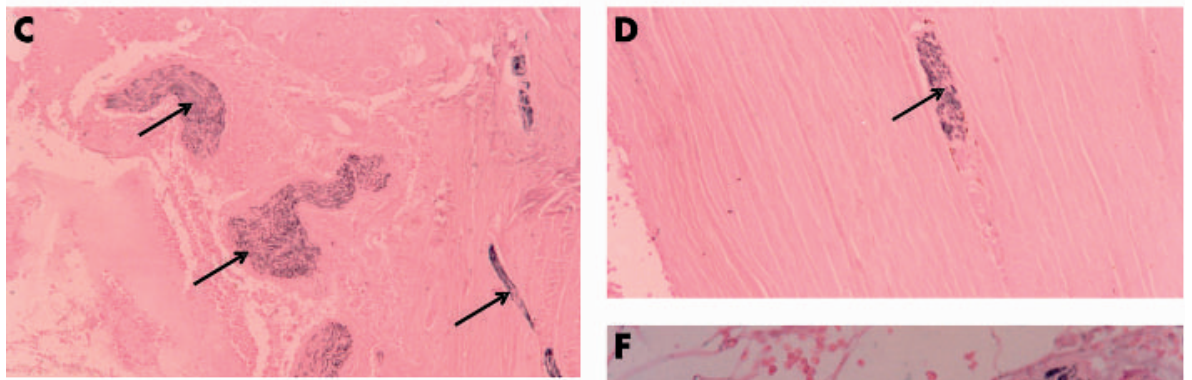

nerves within the sclera

(immunohistochemical stain PGP $9.5 \times 100$ ). (D) Photomicrograph showing intense staining of the posterior ciliary nerves within the sclera (immunohistochemical stain PGP $9.5 \times 100)$. (E) Higher magnification photomicrograph showing intense staining of nerves within the sclera. (immunohistochemical stain PGP $9.5 \times 400)$. (F) Photomicrograph

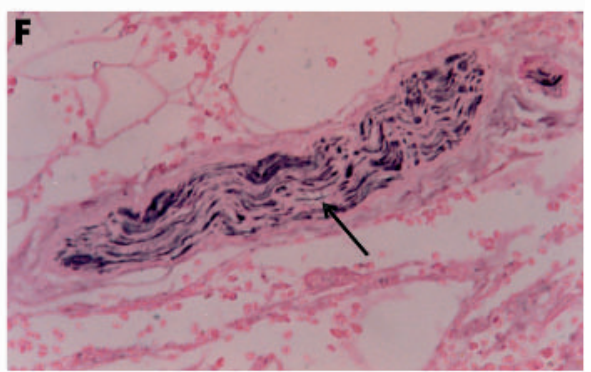
showing intense staining of axons of the posterior ciliary nerves at the level of the optic nerve entry into the eyeball (immunohistochemical stain PGP $9.5 \times 400)$.

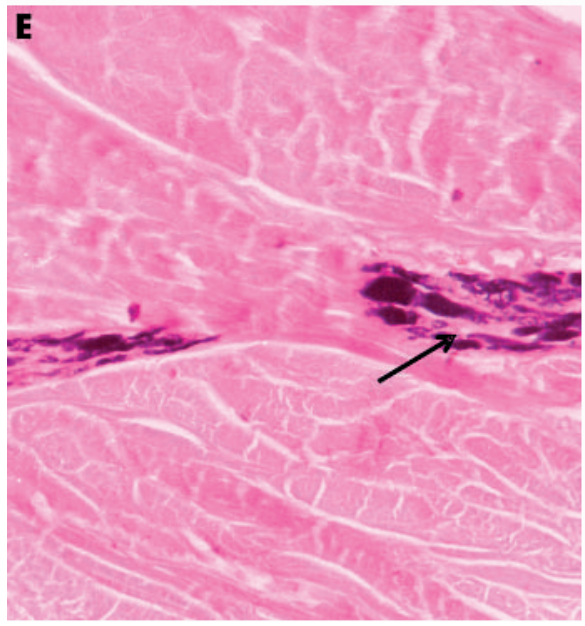

staining of the nerves in the ciliary processes and the ciliary body (fig $1 \mathrm{~B}$ ). The ciliary nerves running within the sclera were also intensely stained with the PGP 9.5 (fig 1C, D, and E). The posterior ciliary nerves showed intense axonal staining with PGP 9.5 at its course near the optic nerve just beyond the posterior pole of the globe (fig lF).

\section{Leprosy eye I}

Corneal epithelium showed diffuse atrophy and the stroma had large granulomas consisting of foamy macrophages, lymphocytes, and plasma cells. Foci of scarring with calcium precipitations were seen below the corneal epithelium. Scattered macrophages were seen throughout the corneal stroma. Extensive diffuse infiltration was seen near the limbus with focal pannus formation. Parts of the iris were seen adhering to the inferior part of the corneal stroma. Iris and ciliary body showed extensive diffuse dense inflammatory infiltrates composed of macrophages, lymphocytes, and plasma cells.

There was extensive destruction of the ciliary muscles. Collections of foamy macrophages were seen on the anterior segment of the lamina fusca layer of the choroid. A nerve bundle in the sclera at the level of the muscle insertion showed perineurial lymphocytic infiltrates, perineurial and intraneural fibrosis, but did not show any intraneural lymphocytic infiltrate. Small collections of macrophages and dilated blood vessels were seen on the anterior segment of sclera up to the insertion of recti muscles.

Acid fast stained sections showed few bacillus on the corneal and conjunctival epithelium and in the corneal stroma. Clumps of bacilli were seen within the macrophage granulomas infiltrating the corneal stroma, sclera, iris, and ciliary body (fig 2A). Chorio-capillaries situated anteriorly demonstrated bacilli. Bacilli were also seen within macrophages, nerves, and walls of blood vessels present in the anterior part of choroid and sclera, up to the insertion of the external ocular rectus muscles. Solochrome cyanine stain showed complete absence of myelin in the posterior ciliary nerves. PGP 9.5 stain (Pan axonal marker) showed complete absence of axonal staining in the posterior ciliary nerves lying on the temporal side of the optic nerve. The ciliary nerves running within the scleral tissue and the nerve twigs in the ciliary muscle also showed complete absence of staining with PGP 9.5.

\section{Leprosy eye II}

The histopathology was similar. PGP 9.5 staining showed absence of staining of the nerve endings of the ciliary muscle (fig 2B), mild patchy staining of the posterior ciliary nerves running within the scleral tissue (fig 2C), and absence of staining in the ciliary nerves on the temporal side of the optic nerve (fig 2D, E). 

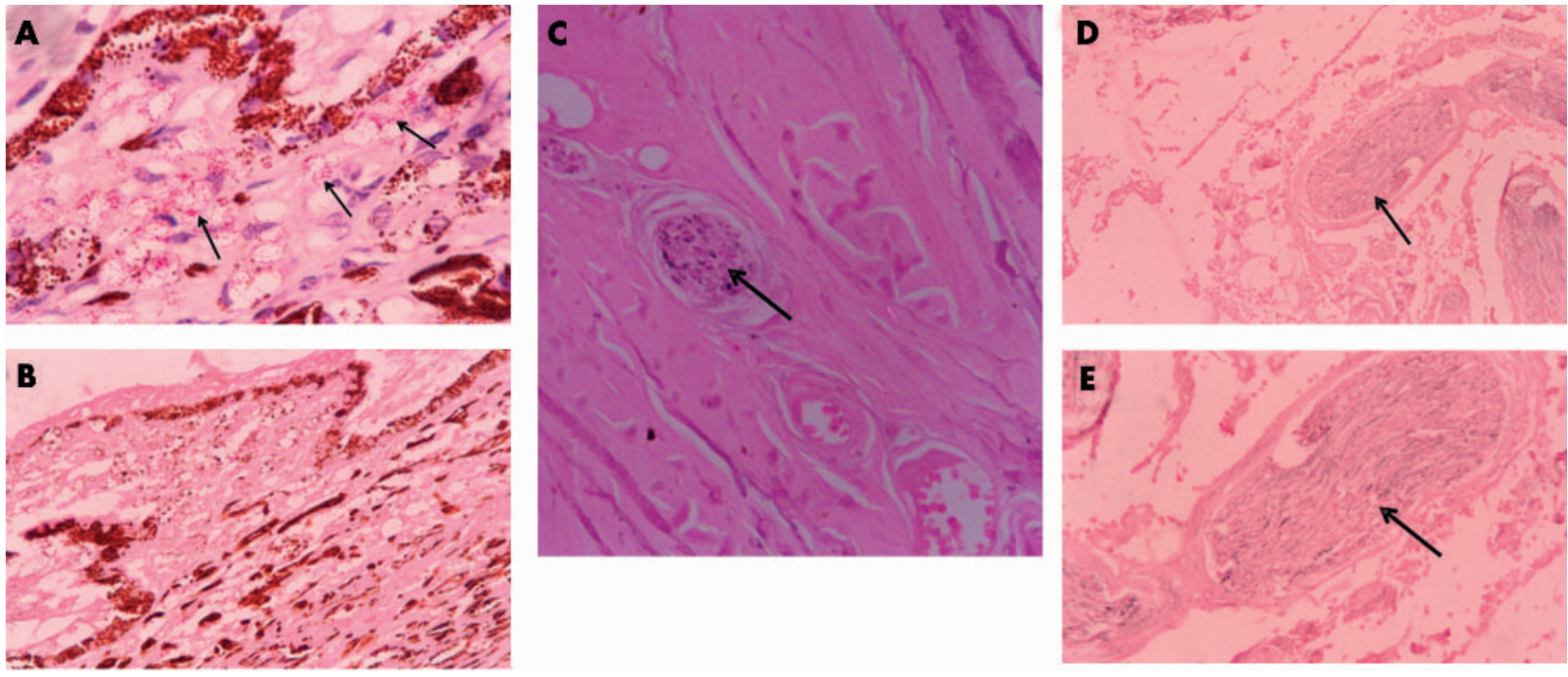

Figure 2 Leprosy eyes, histopathology. (A) Photomicrograph showing ciliary process of the lepromatous eye infiltrated by macrophages containing Mycobacterium leprae (acid fast stain $\times 400$ ). (B) Photomicrograph of ciliary process showing complete absence of staining of ciliary nerve endings (immunohistochemical stain PGP $9.5 \times 400$ ). (C) Photomicrograph of sclera showing poor and patchy staining of a posterior ciliary nerve within the sclera (immunohistochemical stain PGP 9.5×400). (D) Photomicrograph of posterior ciliary nerve situated beside the optic nerve at its site of entry into the eyeball, showing complete absence of axonal staining (immunohistochemical stain PGP 9.5 $\times 400$ ). (E) Magnified photomicrograph of posterior ciliary nerve situated beside the optic nerve at its site of entry into the eyeball, showing complete absence of axonal staining (immunohistochemical stain PGP $9.5 \times 400)$.

\section{DISCUSSION}

Protein gene product (PGP) 9.5 is a neuron specific protein with a molecular weight of $27 \mathrm{kD}$. Standard immunohistochemical techniques have shown the presence of PGP 9.5 in neurons and nerve fibres at all levels of the central and peripheral nervous system, in neuroendocrine cells, in renal tubules, in spermatogonia and Leydig cells of the testis, and in the corpus luteum. PGP 9.5 is of value as a marker for neurons and peripheral nerve fibres and has been found to be of particular use in the study of ubiquitinated cellular inclusions characteristic of several chronic human neurodegenerative diseases. ${ }^{7-9}$ PGP 9.5 has been used in studying several types of tumours ${ }^{10-12}$ and in staining ocular tissues. ${ }^{13-17}$

The posterior ciliary nerves are formed by the confluence of the sympathetic, sensory, and post synaptic parasympathetic axons. They lie in the outer choroid and branch near the ora serrata to form a rich plexus of myelinated and unmyelinated nerves which supply the iris and the ciliary body. ${ }^{18}$ In the non-leprosy eyes, after staining with PGP 9.5, several stained nerve bundles within the ciliary body were easily visible. Even after protracted search in several slides, nerves or nerve endings of the ciliary body, stained with PGP 9.5, were hardly discernable in the two lepromatous eyes, indicating extensive destruction of the ciliary body nerves.

An important finding in this study is the occurrence of loss of axonal staining with PGP 9.5, signifying disruption of axonal fibres of the posterior ciliary nerves, even at the level just beyond the posterior pole of the eye, lateral to the optic nerve. This finding was present in both the lepromatous eyes. In the non-lepromatous eyes, the posterior ciliary nerve axons stained well with the PGP 9.5 stain, indicating intact nerves. Following up these posterior ciliary nerves into the scleral layer we were able to show, in certain sections, that these nerves contained within the scleral sheath continued to stain well in the non-lepromatous eyes while in the lepromatous eyes, no stain was taken up. There was no localised inflammatory histopathology surrounding the posterior ciliary nerves at the posterior pole of the lepromatous eyes to account for nerve destruction at that site. Mycobacterium leprae were also not present anywhere near the vicinity of these nerves. It therefore seems plausible that the axonopathy of the posterior ciliary nerves is a result of a "dying back" phenomenon.

Lepromatous leprosy is characterised by infection with millions of Mycobacterium leprae that have a predilection for Schwann cells and perineural cells of peripheral cutaneous nerves. The neuritis that follows eventually destroys the nerves. In many lepromatous patients a type of anaesthesia called the "glove and stocking", where there is symmetrical loss of sensation in all four limbs due to an ascending polyneuritis of the extremities, occurs. ${ }^{19}$ We equate this phenomenon in the limbs to what we have shown histopathologically in the eyes of these two lepromatous patients, where an inflammatory process with the presence of $M$ leprae confined to the anterior segment of the globe extends the neuronal damage well beyond the posterior pole of the globe through the posterior ciliary nerves, by an ascending neuritis. The posterior ciliary nerves not only carry sensory fibres to the ciliary body and iris, but are also the parasympathetic and sympathetic supply to these tissues. They also supply sensory fibres to the cornea. Therefore, destruction to the posterior ciliary nerves may, in part, explain the corneal sensory impairment and the miotic pupil that are features often encountered in long standing lepromatous leprosy patients.

\section{ACKNOWLEDGEMENTS}

We are grateful to Professor Justin C McArthur, Deputy Director, Department of Neurology, Johns Hopkins University, USA for his advice and encouragement. We also thank the American Leprosy Missions, Greenville, South Carolina, USA, for their financial support and Mr Sekar and Professor Charles K Job for their input.

\section{Authors' affiliations}

G J Ebenezer, Head, Department of Histopathology and Experiment Pathology, Schieffelin Leprosy Research and Training Center, Karigiri, Vellore District, Tamilnadu, India

E Daniel, Head, Department of Ophthalmology, Schieffelin Leprosy Research and training Center, Karigiri, Vellore District, Tamilnadu, India 632106 
Correspondence to: Dr Ebenezer Daniel, Head, Department of Ophthalmology, Schieffelin Leprosy Research and Training Center, Karigiri, Vellore District, Tamilnadu, India - 632106; oculep@vsnl.com

Accepted for publication 10 June 2003

\section{REFERENCES}

1 Lockwood DNJ. Leprosy elimination - a virtual phenomenon or a reality? BMJ 2002:324:1516-18.

2 Daniel E, Ebenezer GJ, Job CK. Pathology of iris in leprosy. Br J Ophthalmol 1997;81:490-2.

3 Job CK, Ebenezer GJ, Thompson K, et al. Pathology of eye in leprosy. Indian J Lepr 1998;70:79-92.

4 Daniel E, Ebenezer GJ. Pathology of a lepromatous eye. Int J Lepr Other Mycobact Dis 2000;68:23-6.

5 Daniel E, Ebenezer GJ, Ffytche TJ, et al. Epithelioid granuloma in the iris of a lepromatous leprosy patient: an unusual finding. Int $J$ Lepr Other Mycobact Dis 2000;68:152-4.

6 McArthur JC, Stocks AE, Hauer $P$, et al. Epidermal nerve fiber densitynormative reference range and diagnostic efficiency. Arch Neurol 1998:55:1513-20.

7 Lowe J, McDermott $\mathrm{H}$, Landon $\mathrm{M}$, et al. Ubiquitin carboxyl-terminal hydrolase (PGP 9.5) is selectively present in ubiquitinated inclusion bodies characteristic of human neurodegenerative diseases. J Pathol 1990;161:153-60.
8 Wilkinson KD, Lee K, Deshpande S, et al. The neuron-specific protein PGP 9.5 is a ubiquitin carboxyl-terminal hydrolase. Science 1989;246:670-5.

9 Thompson RJ, Doran JF, Jackson P, et al. PGP 9.5- a new marker for vertebrate neurons and neuronendocrine cells. Brain Res 1983;278:224-8.

10 Hibi K, Westra WH, Borges M, et al. PGP9.5 as a candidate tumor marker for non-small-cell lung cancer. Am J Pathol 1999;155:711-5.

11 Furuno K, Wakakura M, Shimizu K, et al. Immunohistochemical studies of Merkel cell carcinoma of the eyelid. Jpn J Ophthalmol 1992;36:348-55.

12 Tezel E, Hibi K, Nagasaka T, et al. PGP9.5 as a prognostic factor in pancreatic cancer. Clin Cancer Res 2000;6:4764-7.

13 Gavazzi I, Canavan RE, Cowen T. Influence of age and anti-nerve growth factor treatment on the sympathetic and sensory innervation of the rat iris. Neuroscience 1996;73:1069-79.

14 Marfurt CF, Jones MA, Thrasher K. Parasympathetic innervation of the rat cornea. Exp Eye Res 1998;66:437-48.

15 Rios JD, Forde K, Diebold Y, et al. Development of conjunctival goblet cells and their neuroreceptor subtype expression. Invest Ophthalmol Vis Sci 2000;41:2127-37.

16 Marfurt CF, Murphy CJ, Florczak JL. Morphology and neurochemistry of canine corneal innervation. Invest Ophthalmol Vis Sci 2001;42:2242-51.

17 Nakajima J, Mekada A, Nakamura J, et al. Expression of protein gene product 9.5 in the anterior lens epithelial cells of atopic cataracts. J Cataract Refract Surg 2002; 28:2035-9.

18 Bron AJ, Tripathi RC, Tripathi BJ. Wolff's. In: Anatomy of the Eye and the orbit: The Ciliary Body. 8th ed. London UK: Chapman and Hall, 1977:360-1.

19 Weddell G. Disorders of peripheral cutaneous nerves. J Invest Dermatol 1977:69:130-5.

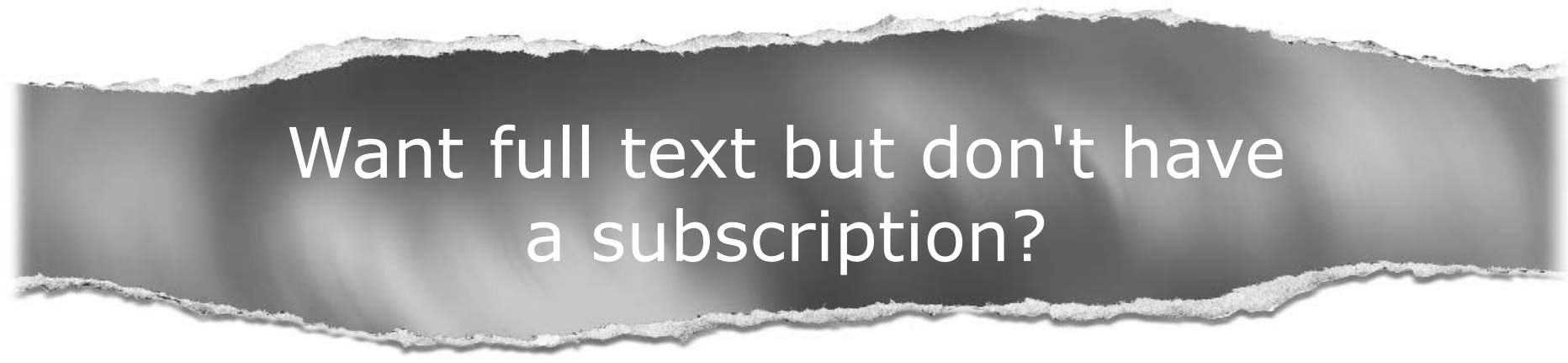

\section{Pay per view}

For just $\$ 8$ you can purchase the full text of individual articles using our secure online ordering service. You will have access to the full text of the relevant article for 48 hours during which time you may

download and print the pdf file for personal use.

\section{www.bjophthalmol.com}

